\title{
HIGH ENERGY GAMMA-RAY EMISSION FROM BLAZARS:
}

\author{
EGRET OBSERVATIONS*
}

\author{
R. MUKHERJEE \\ Barnard College $\&$ Columbia University \\ Dept. of Physics 8 Astronomy \\ 3009 Broadway, 506 Altschul \\ New York, NY 10027
}

\begin{abstract}
We will present a summary of the observations of blazars by the Energetic Gamma Ray Experiment Telescope (EGRET) on the Compton Gamma Ray Observatory (CGRO). EGRET has detected high energy $\gamma$ ray emission at energies greater than $100 \mathrm{MeV}$ from more that 50 blazars. These sources show inferred isotropic luminosities as large as $3 \times 10^{49}$ ergs $\mathrm{s}^{-1}$. One of the most remarkable characteristics of the EGRET observations is that the $\gamma$-ray luminosity often dominates the bolometric power of the blazar. A few of the blazars are seen to exhibit variability on very short time-scales of one day or less. The combination of high luminosities and time variations seen in the $\gamma$-ray data indicate that $\gamma$-rays are an important component of the relativistic jet thought to characterize blazars. Currently most models for blazars involve a beaming scenario. In leptonic models, where electrons are the primary accelerated particles, $\gamma$-ray emission is believed to be due to inverse Compton scattering of low energy photons, although opinions differ as to the source of the soft photons. Hardronic models involve secondary production or photomeson production followed by pair cascades, and predict associated neutrino production.
\end{abstract}

\section{Introduction}

One of the most striking accomplishments of the Energetic Gamma Ray Experiment Telescope (EGRET) instrument on the Compton Gamma-Ray Observatory (CGRO) is the detection of high-energy $\gamma$-rays from active galaxies whose emission at most wavebands is dominated by non-thermal

\footnotetext{
*Invited review paper to appear in Observational Evidence for Black Holes in the Universe, ed. S. K. Chakrabarti, 1999 (Dordrecht: Kluwer) pg 215-230.
} 
processes. These objects, called "blazars," are highly variable at most frequencies and are bright radio sources. Prior to the launch of CGRO, 3C 273, discovered by COS-B (Swanenburg et al. 1978), was the only known extragalactic source of $\gamma$-rays. Since then, EGRET has detected more than 50 blazars in high energy $(>100 \mathrm{MeV}) \gamma$-rays (Mukherjee et al. 1997; Thompson et al. 1995; 1996).

The blazars detected by EGRET all share the common characteristic that they are radio-loud, flat-spectrum radio sources, with radio spectral indices $\alpha_{r} \geq-0.6$ (von Montigny et al. 1995). Several of these blazars are known to demonstrate superluminal motion of components resolved with VLBI (3C 279, 3C 273, 3C 454.3, PKS 0528+134, for example). The blazar class of active galactic nuclei (AGN) includes BL Lac objects, highly polarized quasars (HPQ), or optically violent variable (OVV) quasars and are characterized by one or more of the properties of this source class, namely, a non-thermal continuum spectrum, a flat radio spectrum, strong variability and optical polarization. For many of the EGRET-detected blazars, the $\gamma$-ray energy flux is dominant over the flux in lower energy bands. The redshifts of these sources range from 0.03 to 2.28 and the average photon spectral index, assuming a simple power law fit to the spectrum, is $\sim 2.2$. Many of the blazars exhibit variability in their $\gamma$-ray flux on timescales of several days to months. In addition, blazars exhibit strong and rapid variability in both optical and radio wavelengths.

Of the 51 blazars reviewed here, 14 are BL Lac objects, and the rest are flat spectrum radio quasars (FSRQs). BL Lac objects generally have stronger polarization and weaker optical lines. In fact, some BL Lac objects have no redshift determination because they have no identified lines above their optical continuum. FSRQs are generally more distant and more luminous compared to the BL Lac objects.

This review summarizes the present knowledge on $\gamma$-ray observations of blazars by EGRET. A brief description of the EGRET instrument and data analysis techniques, and the list of blazars detected by EGRET is given in $\S 2$. Temporal variations and $\gamma$-ray luminosity of blazars are discussed in $\S \S 3 \& 4$. Section 5 describes the spectral energy distribution of blazars and summarizes the various models that have been proposed to explain the $\gamma$-ray emission in blazars.

\section{EGRET observations and analysis}

\subsection{THE EGRET INSTRUMENT}

EGRET is a $\gamma$-ray telescope that is sensitive in the energy range $\sim 30 \mathrm{MeV}$ to $30 \mathrm{GeV}$. It has the standard components of a high-energy $\gamma$-ray instrument: an anticoincidence dome to discriminate against charged particles, 
a spark chamber particle track detector with interspersed high- $Z$ material to convert the $\gamma$-rays into electron-positron pairs, a triggering telescope to detect the presence of the pair with the correct direction of motion, and an energy measurement system, which in the case of EGRET is a $\mathrm{NaI}(\mathrm{Tl})$ crystal. EGRET has an effective area of $1500 \mathrm{~cm}^{2}$ in the energy range 0.2 $\mathrm{GeV}$ to $1 \mathrm{GeV}$, decreasing to about one-half the on-axis value at $18^{\circ}$ off-axis and to one-sixth at $30^{\circ}$. The instrument is described in details by Hughes et al. (1980) and Kanbach et al. $(1988,1989)$ and the preflight and postflight calibrations are given by Thompson et al. (1993) and Esposito et al. (1998), respectively.

Although EGRET records individual photons in the energy range 30 $\mathrm{MeV}$ to about $30 \mathrm{GeV}$, there are several instrumental characteristics that limit the energy range for which time variation investigations of blazars are viable. At the low end of the energy range, below $\sim 70 \mathrm{MeV}$, there are systematic uncertainties that make the spectral information marginally useful. In addition, the deteriorating point spread function (PSF) and energy resolution at low energies, make analysis more difficult. At high energies, although the systematic uncertainties are reduced, and the PSF and energy resolution are more reasonable, because of the steeply falling spectra, few photons are detected above $5 \mathrm{GeV}$.

The angular resolution of EGRET is energy dependent, varying from about $8^{\circ}$ at $60 \mathrm{MeV}$ to $0.4^{\circ}$ above $3 \mathrm{GeV}$ (68\% containment). The positions of sources are detected with varying accuracy: better than $0.1^{\circ}$ for the very bright sources, or at least $0.5^{\circ}$ for sources just above the detection threshold.

The threshold sensitivity of EGRET ( $>100 \mathrm{MeV}$ ) for a single observation is $\sim 3 \times 10^{-7}$ photons $\mathrm{cm}^{-2} \mathrm{~s}^{-1}$, and is only about a factor of 50-100 below the maximum blazar flux ever observed. The dynamic range for most observations of blazar variations is, therefore, fairly small.

\subsection{EGRET DATA ANALYSIS}

The blazars described here were typically observed by EGRET for a period of 1 to 2 weeks; however, several of them were observed for 3 to 5.5 weeks. Following the standard EGRET processing of individual $\gamma$-ray events, summary event files were produced with $\gamma$-ray arrival times, directions and energies. For the observations reported here, photons coming from directions greater than $30^{\circ}$ from the center of the field of view (FOV) were not used, in order to restrict the analysis to photons with the best energy and position determinations. In addition, exposure history files were produced containing information on the instrument's mode of operation and pointing. These maps were used to generate skymaps of counts and intensity for the entire field of view for each observation, using a grid of $0.5^{\circ} \times 0.5^{\circ}$. The 
intensity maps were derived simply by dividing the counts by the exposure. The EGRET data processing techniques are described further by Bertsch et al. (1989).

The number of source photons, distributed according to the instrument PSF in excess of the diffuse background, was optimized. An $E^{-2}$ photon spectrum was initially assumed for the source search. The background diffuse radiation was taken to be a combination of a Galactic component caused by cosmic ray interactions in atomic and molecular hydrogen gas (Hunter et al. 1997), as well as an almost uniformly distributed component that is believed to be of extragalactic origin (Sreekumar et al. 1998).

The data were analyzed using the method of maximum likelihood as described by Mattox et al. (1996) and Esposito et al. (1998). The likelihood value, $L$, for a model of the number of $\gamma$-rays in each pixel of a region of the map is given by the product of the probability that the measured counts are consistent with the model counts assuming a Poisson distribution. The probability of one model with likelihood, $L_{1}$, better representing the data than another model with likelihood, $L_{2}$, is determined from twice the difference of the logarithms of the likelihoods, $2\left(\ln L_{2}-\ln L_{1}\right)$. This difference, referred to as the test statistic $T S$, is distributed like $\chi^{2}$ with the number of degrees of freedom being the difference in the number of free parameters in the two models. The flux of the point source and the flux of the diffuse background emission in the model are adjusted to maximize the likelihood. The significance of a source detection in sigma is given approximately by the square root of $T S$.

\subsection{EGRET OBSERVATIONS}

The 51 blazars listed in Table 1 were all detected by EGRET above 100 $\mathrm{MeV}$ during the period of EGRET observations from 1991 April to 1995 September (Phases 1 through 4 of CGRO) (Mukherjee et al. 1997). Some of these blazar associations are not certain; Mattox et al. (1997a) find only 42 identifications to have high confidence. Conversely, some of the unidentified high-latitude EGRET sources are likely to be blazars. In addition to the 42 considered strongest, Mattox et al. (1997a) note 16 possible associations with bright flat-spectrum, blazar-like radio sources. Typically, each blazar listed in Table 1 was seen in several different viewing periods (VPs). The maximum and minimum fluxes observed for each blazar is indicated in Table 1. A more complete list of blazar detections by EGRET may be found in the third EGRET catalog (Hartman et al. 1998). 
TABLE 1

EGRET-DETECTED BLAZARS

\begin{tabular}{|c|c|c|c|c|c|c|}
\hline Name & $\begin{array}{l}\text { Other } \\
\text { Names }\end{array}$ & $\mathrm{z}^{\mathrm{a}}$ & $\begin{array}{c}\text { Maximum }^{\mathrm{b}} \\
\text { Flux }\end{array}$ & $\begin{array}{l}\text { Minimum }^{\mathrm{b}} \\
\text { Flux }\end{array}$ & $\begin{array}{l}\text { Source } \\
\text { Type }\end{array}$ & $\mathrm{V}^{\mathrm{c}}$ \\
\hline $0202+149$ & \multicolumn{2}{|l|}{$4 \mathrm{C}+15.05$} & $24.5 \pm 10.7$ & $<9$ & FSRQ & 2.29 \\
\hline 0208-512 & & 1.003 & $131.9 \pm 24.7$ & $15.9 \pm 8.7$ & FSRQ & 11.38 \\
\hline $0219+428$ & $3 \mathrm{C} 66 \mathrm{~A}$ & 0.444 & $25.5 \pm 5.8$ & $<17$ & $\mathrm{BL} \mathrm{LAC}$ & 1.05 \\
\hline $0235+164$ & OD160 & 0.94 & $82.8 \pm 9.2$ & $<24$ & BL LAC & 7.96 \\
\hline 0336-019 & CTA26 & 0.852 & $186.2 \pm 7.6$ & $<15$ & FSRQ & 5.48 \\
\hline 0420-014 & OA 129 & 0.915 & $51.2 \pm 10.5$ & $<13$ & FSRQ & 2.14 \\
\hline 0440-003 & \multirow[t]{9}{*}{ NRAO 190} & 0.844 & $84.4 \pm 12.0$ & $<11$ & FSRQ & 5.05 \\
\hline $0446+112$ & & 1.207 & $105.4 \pm 19.2$ & $<12$ & FSRQ & 2.72 \\
\hline 0458-020 & & 2.286 & $30.8 \pm 9.5$ & $<11$ & FSRQ & 0.47 \\
\hline $0521-365$ & & 0.055 & $37.5 \pm 11.2$ & $<13$ & BL LAC & 2.04 \\
\hline $0528+134$ & & 2.06 & $307.6 \pm 34.6$ & $<40$ & FSRQ & $>15$ \\
\hline 0537-441 & & \multirow[t]{2}{*}{0.894} & $89.8 \pm 14.5$ & $<18$ & BL LAC & 4.43 \\
\hline $0716+714$ & & & $44.0 \pm 11.0$ & $<35$ & BL LAC & 1.21 \\
\hline $0735+178$ & & $>.424$ & $40.9 \pm 21.3$ & $<17$ & BL LAC & 0.30 \\
\hline $0827+243$ & & 2.046 & $68.1 \pm 14.4$ & $<26$ & FSRQ & 2.00 \\
\hline $0829+046$ & OJ+49 & 0.18 & $19.1 \pm 10.6$ & $<30$ & BL LAC & 0.11 \\
\hline $0836+710$ & \multirow[t]{2}{*}{$4 \mathrm{C}+71$} & 2.17 & $31.4 \pm 8.9$ & $<7$ & FSRQ & 1.92 \\
\hline $0917+449$ & & 2.18 & $32.1 \pm 9.8$ & $<31$ & FSRQ & 0.29 \\
\hline $0954+556$ & \multirow[t]{2}{*}{$4 \mathrm{C}+55$} & 0.901 & $48.1 \pm 15.7$ & $<11$ & FSRQ & 0.61 \\
\hline $0954+658$ & & 0.368 & $17.8 \pm 9.4$ & $<5$ & BL LAC & 1.24 \\
\hline $1101+384$ & Mrk 421 & 0.031 & $26.2 \pm 6.9$ & $<16$ & BL LAC & 0.56 \\
\hline $1127-145$ & OM-146 & 1.187 & $80.6 \pm 19.9$ & $<16$ & FSRQ & 1.69 \\
\hline $1156+295$ & $4 \mathrm{C}+29$ & 0.729 & $192.2 \pm 47.8$ & $<6$ & FSRQ & 3.01 \\
\hline $1219+285$ & ON231 & 0.102 & $12.7 \pm 6.8$ & $<11$ & $\mathrm{BL} \mathrm{LAC}$ & 0.92 \\
\hline $1222+216$ & $4 \mathrm{C} 21.35$ & 0.435 & $50.7 \pm 15.6$ & $<8$ & FSRQ & 3.42 \\
\hline $1226+023$ & \multirow[t]{2}{*}{$3 \mathrm{C} 273$} & 0.158 & $55.7 \pm 11.9$ & $<11$ & FSRQ & 3.48 \\
\hline $1229-021$ & & 1.0448 & $13.4 \pm 4.0$ & $<8$ & FSRQ & 1.10 \\
\hline $1253-055$ & $3 \mathrm{C} 279$ & 0.538 & $287.8 \pm 10.9$ & $<18$ & FSRQ & $>15$ \\
\hline $1331+170$ & $\mathrm{OP}+151$ & 2.0838 & $12.8 \pm 8.1$ & $<6$ & FSRQ & 0.22 \\
\hline $1406-076$ & \multirow[t]{2}{*}{ OQ-010 } & 1.494 & $127.6 \pm 23.4$ & $<10$ & FSRQ & $>15$ \\
\hline $1424-418$ & & 1.522 & $31.5 \pm 9.9$ & $<13$ & FSRQ & 1.26 \\
\hline $1510-089$ & OR-017 & 0.361 & $28.6 \pm 7.6$ & $<28$ & FSRQ & 0.30 \\
\hline $1604+159$ & $4 \mathrm{C}+15.54$ & 0.357 & $41.4 \pm 12.2$ & $<18$ & BL LAC & 0.84 \\
\hline $1606+106$ & $4 \mathrm{C}+10.45$ & 1.24 & $62.5 \pm 13.0$ & $<15$ & FSRQ & 3.34 \\
\hline $1611+343$ & \multirow[t]{3}{*}{ OS +319} & 1.404 & $73.2 \pm 15.6$ & $<10$ & FSRQ & 7.05 \\
\hline $1622-253$ & & 0.786 & $60.6 \pm 22.3$ & $<38$ & FSRQ & 0.34 \\
\hline $1622-297$ & & 0.815 & $245.6 \pm 31.8$ & $<15$ & FSRQ & $>15$ \\
\hline $1633+382$ & \multirow[t]{2}{*}{ 4C 38.41} & 1.810 & $98.3 \pm 9.3$ & $<88$ & FSRQ & 4.72 \\
\hline $1730-130$ & & 0.902 & $120.2 \pm 36.5$ & $<42$ & FSRQ & 1.79 \\
\hline $1739+522$ & \multirow[t]{4}{*}{$4 \mathrm{C}+51.37$} & \multirow[t]{2}{*}{1.375} & $51.0 \pm 27.6$ & $<29$ & FSRQ & 1.03 \\
\hline 1908-021 & & & $43.7 \pm 27.5$ & $<14$ & FSRQ & 0.81 \\
\hline $1933-400$ & & \multirow[t]{2}{*}{0.966} & $99.4 \pm 32.2$ & $<9$ & FSRQ & 1.78 \\
\hline $2022-077$ & & & $72.0 \pm 13.3$ & $<5$ & FSRQ & 3.51 \\
\hline $2032+107$ & \multirow[t]{3}{*}{$\mathrm{OW}+154$} & 0.601 & $24.4 \pm 10.2$ & $<12$ & $\mathrm{BL} \mathrm{LAC}$ & 0.20 \\
\hline $2052-474$ & & 1.489 & $37.6 \pm 21.6$ & $<11$ & FSRQ & 1.58 \\
\hline $2155-304$ & & 0.116 & $32.3 \pm 7.8$ & $<17$ & BL LAC & 1.77 \\
\hline $2200+420$ & \multirow[t]{2}{*}{ BL Lac } & \multirow[t]{2}{*}{0.069} & $78.1 \pm 38.3^{\mathrm{d}}$ & $<16$ & BL LAC & $0.69^{\mathrm{d}}$ \\
\hline $2209+236$ & & & $35.8 \pm 18.0$ & $<10$ & FSRQ & 0.43 \\
\hline $2230+114$ & CTA 102 & 1.037 & $38.8 \pm 13.9$ & $<27$ & FSRQ & 1.79 \\
\hline $2251+138$ & $3 \mathrm{C} 454.3$ & 0.859 & $119.3 \pm 18.7$ & $<50$ & FSRQ & 3.21 \\
\hline $2356+196$ & $\mathrm{OZ}+193$ & 1.066 & $29.7 \pm 9.0$ & $<17$ & FSRQ & 0.87 \\
\hline $\begin{array}{l}\text { a Redshift } \\
\text { b Units: } 1 \\
\text { c Variabil } \\
\text { d Both th } \\
\text { aken into a }\end{array}$ & $\begin{array}{l}\text { h } \mathrm{cm}^{-2} \mathrm{~s} \\
\text { dex (see te } \\
\text { timum flux } \\
\text { (Bloom }\end{array}$ & 1997). & MTE & 政 & 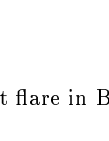 & e \\
\hline
\end{tabular}



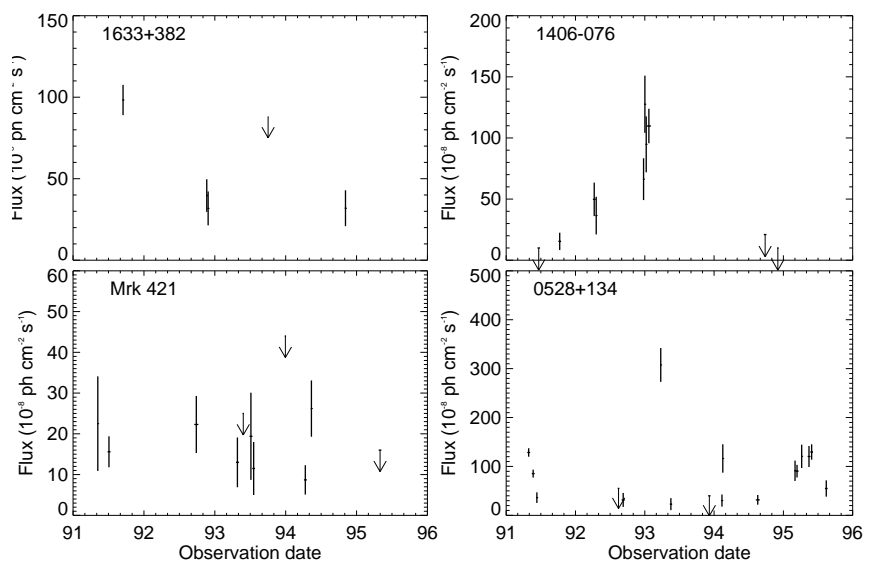

Figure 1. Flux history of four blazars (PKS 1633+382, PKS 1406-076, Mrk 421, and PKS 0528+134) from 1991 April to 1995 September, as detected by EGRET. $2 \sigma$ upper limits are indicated by downward arrows.

\section{Time variability}

The fluxes of the blazars detected by EGRET have been found to be variable on time scales of a year or more down to well under a day. Long term variations of blazars have been addressed earlier by several authors (eg. von Montigny et al. 1995; Hartman et al. 1996a; Mukherjee et al. 1997). In some cases, many of the detected blazars have exhibited flux variations up to a factor of about 30 between different observations. Figure 1 shows the flux history of four EGRET-detected blazars. The horizontal bars on the individual data points denote the extent of the VP for that observation. Fluxes have been plotted for all detections greater than $2 \sigma$. For detections below $2 \sigma$, upper limits at the $95 \%$ confidence level are shown. A systematic uncertainty of $6 \%$ was added in quadrature with the statistical uncertainty for each flux value, consistent with the analysis of McLaughlin et al. (1996) on EGRET source variability.

In order to quantify the flux variability of the blazars in Table 1, Mukherjee et al. (1997) calculated the variability index, $V=\log Q$, as defined by McLaughlin et al. (1996), where $Q=1-P_{\chi}\left(\chi^{2}, \nu\right)$. Here $P_{\chi}\left(\chi^{2}, \nu\right)$ is the probability of observing $\chi^{2}$ or something larger from a $\chi^{2}$ distribution with $\nu$ degrees of freedom. The flux versus time data were fit to a constant flux and the reduced $\chi_{\nu}^{2}$, for $\nu$ degrees of freedom, was calculated using the least square fit method. For a nonvariable source, a constant flux is expected to fit the data well, and the mean value of the $\chi^{2}$ distribution is expected to 


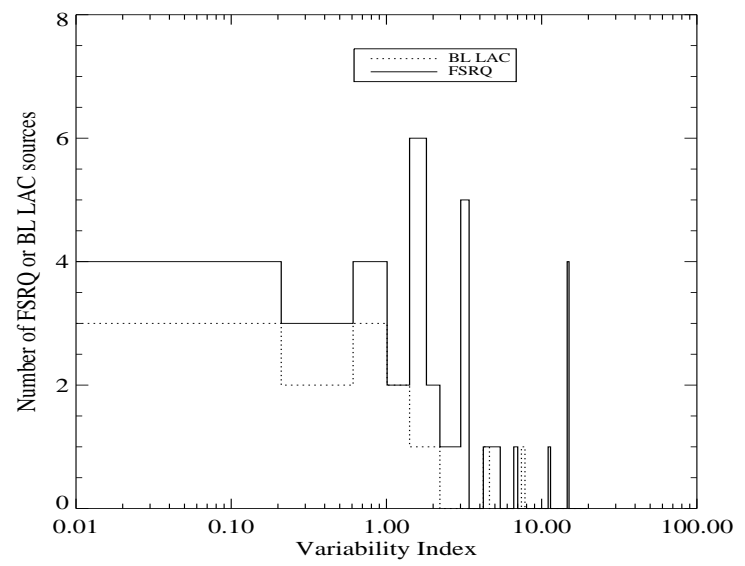

Figure 2. Distributions of the variability indices for BL Lac objects and FSRQs.

be equal to the number of degrees of freedom in the data. The quantity $V$ is used to judge the strength of the evidence for flux variability. Following the classification of McLaughlin et al. , $V<0.5$ was taken to indicate nonvariability, $V \geq 1$ to indicate variability, and $0.5 \leq V<1$ as uncertain. Table 1 lists the value of $V$ for each source.

Of the 51 blazars reviewed here, 35 are found to be variable $(V \geq 1), 9$ are non-variable $(V<0.5)$, and 7 fall in the range of uncertain variability. It should be noted that, although the criterion used here to gauge variability of a blazar is somewhat arbitrary, it does provide a way to compare the numbers obtained. Also, as McLaughlin et al. (1996) have noted, changing these criteria by $20 \%$ yields similar results. If the FSRQs and the BL Lac objects are considered separately, it is found that $76 \%$ of the FSRQs in the sample are variable, while $16 \%$ are non-variable. Similarly, for the BL Lac objects, $50 \%$ are variable, while $21 \%$ are definitely non-variable. It should be noted that the low intrinsic luminosity of BL Lac objects could bias observations (see discussions in $\S 5.2$ ). Figure 2 shows the distribution of the variability indices for the FSRQs and the BL Lac objects. The BL Lac objects in the data set are found to be less variable on the average than the FSRQs. Recent observations of flares in BL Lac objects, however, modify some of these conclusions. For example, BL Lac was detected during a $\gamma$ ray outburst with an average flux of $(171 \pm 42) \times 10^{-8}$ photons $\mathrm{cm}^{-2} \mathrm{~s}^{-1}$ in July 1997 (Bloom et al. 1997). BL Lac would have a high value of $V$ in Table 1, if this information were taken into account.

Figure 3 shows a plot of the variability index as a function of the 


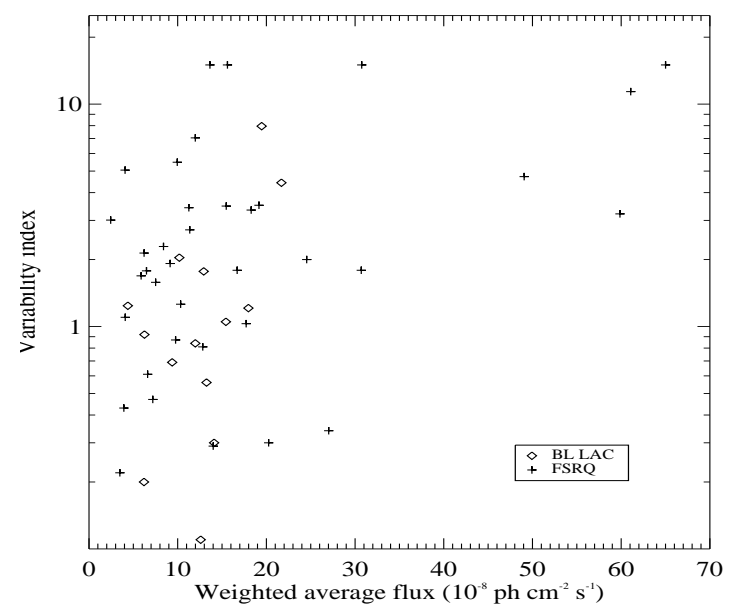

Figure 3. Variability index plotted as a function of the weighted average flux for BL Lac objects and FSRQs. The BL Lac objects are denoted by diamonds and the FSRQs by plus signs in the figure.

weighted average flux for the blazars in Table 1. Note that the sources that have the highest average fluxes all have high variability indices. Only 2 out of 18 blazars with average flux less than $1 \times 10^{-7}$ photons $\mathrm{cm}^{-2} \mathrm{~s}^{-1}$ have a variability index greater than 2.5 . In fact, there are no non-variable blazars in the sample that have high flux.

The study of short term variability in blazars is always limited by the small numbers of photons detected in the short time intervals at $\gamma$-ray energies. The shortest time-scale variations detected for blazars with EGRET are for PKS 1622-297 (Mattox et al. 1997b) and 3C 279 (Wehrle et al. 1997). For both these objects the flux was found to increase by a factor of two or more in less than 8 hours. Other objects that have shown flux variations over the period of a few days are 3C 279 (Kniffen et al. 1993), 3C 454.3 (Hartman et al. 1993), 4C 38.41 (Mattox et al. 1993), PKS 1406-076 (Wagner et al. 1995), and PKS 0528+134 (Hunter et al. 1993; Mukherjee et al. 1996). The short time-scale of $\gamma$-ray flux variability (e.g. in 3C 279 or PKS 1622-297) when combined with the large inferred $\gamma$-ray luminosities, implies that the blazar emission region is very compact. Gamma-ray tests for beaming from variability and flux measurements using the ElliottShapiro relation and $\gamma$-ray transparency arguments are summarized in a recent review on $\gamma$-ray blazars by Hartman et al. (1997). A factor-of-two flux variation on an observed time-scale $\delta t_{\text {obs }}$ limits the size $r$ of a stationary isotropically emitting region to be roughly $r \leq c \delta t_{\mathrm{obs}} /(1+z)$ by simple 
light-travel time arguments. Under the assumptions of isotropic radiation and Eddington-limited accretion, the implied minimum black hole masses of blazars are $\geq 8 \times 10^{11} M_{\odot}$ for PKS 1622-297 (Mattox et al. 1997b) from EGRET observations and $\geq 7.5 \times 10^{8} M_{\odot}$ for PKS $0528+134$ from COMPTEL observations (Collmar et al. 1997).

\section{Luminosity}

The $\gamma$-ray luminosity can be estimated by considering the relationship between the observed differential energy flux $S_{0}\left(E_{0}\right)$, where the subscript "0" denotes the observed or present value, and $Q_{e}$ the power emitted in $d E$, where $E=E_{0}(1+z)$ in the Friedman universe.

$$
Q_{e}\left[E_{0}\right]=4 \pi S_{0}\left(E_{0}\right)(1+z)^{b-1} \Theta D_{L}^{2}\left(z, q_{0}\right)
$$

where

$$
D_{L}=\frac{c}{H_{0} q_{0}^{2}}\left[1-q_{0}+q_{0} z+\left(q_{0}-1\right)\left(2 q_{0} z+1\right)^{1 / 2}\right] \equiv \frac{c z}{H_{0}} g\left(z, q_{0}\right) .
$$

$H_{0}$ is the Hubble parameter, $q_{0}$ is the deceleration parameter, $b$ is the spectral index, $z$ is the redshift, and $\Theta$ is the beaming factor. $H_{0}$ is chosen to be 70 and $q_{0}$ to be 0.5 , although the results obtained here are not highly sensitive to these choices. The beaming factor is taken to be 1 . The spectral index is obtained using the analysis described in $\S 4$. The luminosity as a function of the redshift is determined using equation (1) and is plotted in Figure 4 for the blazars detected by EGRET. The typical detection threshold for EGRET as a function of $z$, for relatively good conditions, is also shown in the figure. The actual threshold varies somewhat with exposure and region of the sky, and the average threshold is a little higher than the curve shown, but the shape is the same. The BL Lac objects are indicated in the figure with dark diamonds, and one sees clearly that they are predominantly closer and lower in luminosity.

Recently, Chiang \& Mukherjee (1998) have calculated the evolution and luminosity function of the EGRET blazars, and have estimated the contribution of this source class to the diffuse extragalactic gamma-ray background. They find that the evolution is consistent with pure luminosity evolution. According to their estimates, only $25 \%$ of the diffuse extragalactic emission measured by SAS-2 and EGRET can be attributed to unresolved $\gamma$-ray blazars, contrary to some of the other estimates (eg. Stecker \& Salamon 1996). Below $10 \mathrm{MeV}$, the average blazar spectrum suggests that only about $50 \%$ of the measured $\gamma$-ray emission could arise from blazars (Sreekumar, Stecker \& Kappadath 1997). This leads to the exciting possibility that other sources of diffuse extragalactic $\gamma$-ray emission must exist. 


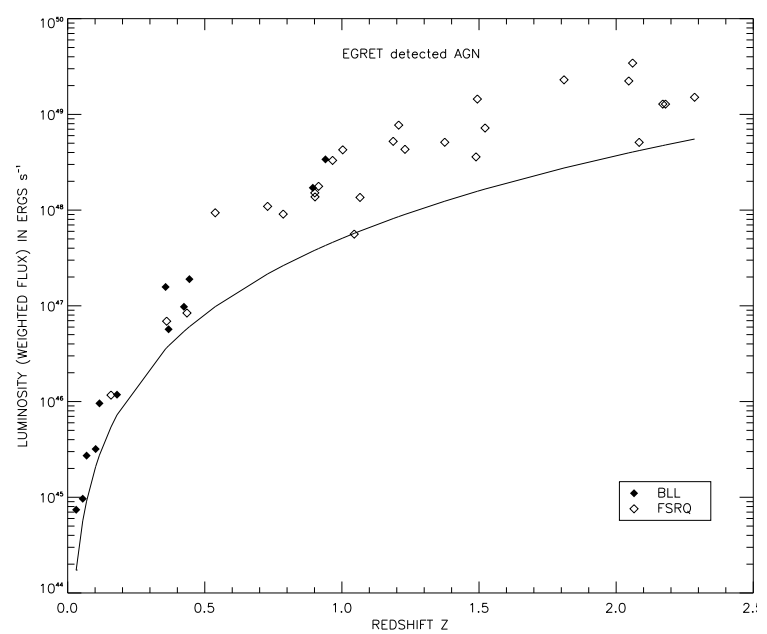

Figure 4. Luminosity vs redshift for blazars detected by EGRET. The BL Lac objects are indicated with filled symbols. The typical detection threshold for EGRET is shown as a solid curve.

\section{Spectra}

\subsection{SPECTRA IN THE EGRET ENERGY RANGE}

EGRET spectra of blazars typically covers at least two decades in energy (from $30 \mathrm{MeV}$ to $10 \mathrm{GeV}$ ) and are well described by a simple power-law model of the form $F(E)=k\left(E / E_{0}\right)^{-\alpha}$ photons $\mathrm{cm}^{-2} \mathrm{~s}^{-1} \mathrm{MeV}^{-1}$, where the photon spectral index, $\alpha$, and the coefficient, $k$, are the free parameters. The energy normalization factor, $E_{0}$, is chosen so that the statistical errors in the power law index and the overall normalization are uncorrelated.

The average blazar spectrum has a spectral index of about -2.15 . Figure 5 shows the photon spectral index of the blazars plotted as a function of the redshift. There are marginal indications that suggest that the BL Lac objects have slightly harder spectrum in the EGRET energy range than the FSRQs. Mukherjee et al. (1997) find that the average spectral index of the BL Lac objects is about -2.03 , compared to about -2.20 for the FSRQs. For some individual blazars there has been noted a trend for the spectrum to harden during a flare state (eg. in blazars 1222+216, 1633+382, and 0528+134; Sreekumar et al. 1996; Mukherjee et al. 1996). A spectral study of blazars as a class has been performed by Mücke et al. (1996) and Pohl et al. (1997). 


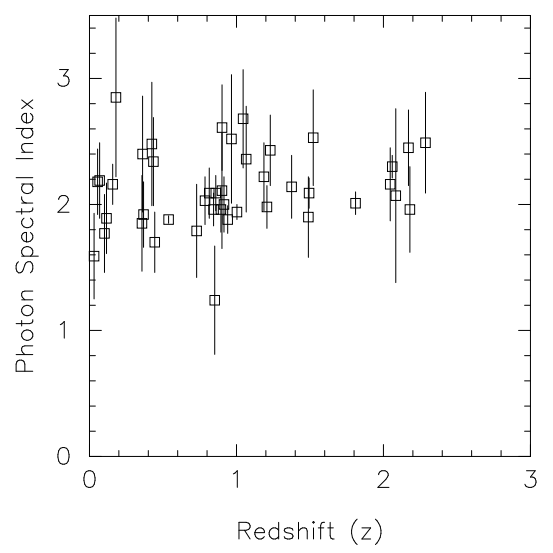

Figure 5. Photon spectral index as a function of the redshift for blazars detected by EGRET.

\subsection{SPECTRAL ENERGY DISTRIBUTIONS AND GAMMA-RAY MODELS}

The processes by which $\gamma$-rays are produced in blazars can be best understood by the study of the correlated multiwaveband observations of blazars extending from radio to $\gamma$-ray wavebands. One of the most significant findings of EGRET is that, in the radio to $\gamma$-ray multiwavelength spectra of blazars, the power in the $\gamma$-ray range equals or exceeds the power in the infrared-optical band. Any model of high-energy $\gamma$-ray emission in blazars needs to explain this basic observational fact. The high $\gamma$-ray luminosity of the blazars suggests that the emission is likely to be beamed and, therefore, Doppler-boosted into the line of sight. This is in agreement with the strong association of EGRET blazars with radio-loud flat-spectrum radio sources, with many of them showing superluminal motion in their jets. This information has helped to favor jet models of emission over models in which the $\gamma$-ray production is directly associated with accretion onto a massive black hole (e.g. Becker \& Kafatos 1993).

The jet models explain the radio to UV continuum from blazars as synchrotron radiation from high energy electrons in a relativistically outflowing jet which has been ejected from an accreting supermassive black hole (Blandford \& Königl 1979). The emission in the $\mathrm{MeV}-\mathrm{GeV}$ range is believed to be due to the inverse Compton scattering of low-energy photons by the same relativistic electrons in the jet. However, two main issues remain questionable: the source of the soft photons that are inverse Compton scattered, and the structure of the inner jet, which cannot be imaged di- 
rectly. The soft photons can originate as synchrotron emission either from within the jet (the synchrotron-self-Compton or SSC process: Maraschi, Ghisellini, \& Celotti 1992; Bloom \& Marscher 1996), or from a nearby accretion disk, or they can be disk radiation reprocessed in broad-emissionline clouds (the external radiation Compton process or the ERC process: Dermer \& Schlickeiser 1994; Sikora, Begelman, \& Rees 1994; Blandford \& Levison 1995; Ghisellini \& Madau 1996). In contrast to these leptonic jet models, the proton-initiated cascade (PIC) model (Mannheim \& Biermann 1989, 1992) predicts that the high-energy emission comes from knots in jets as a consequence of diffusive shock acceleration of protons to energies so high that the threshold of secondary particle production is exceeded.

Figure 6 shows the simultaneous spectral energy distribution of 3C 279 during January-February 1996, when the source was detected at its highest state ever (Wehrle et al. 1997). The figure shows the relative amounts of energy detected in equal logarithmic frequency bands. The power output in $\gamma$-rays dominates the bolometric luminosity of the sources, as mentioned in $\S 1$. Wehrle et al. (1997) note that the $\gamma$-rays vary by more than the square of the observed IR-optical flux change, a fact that could be hard to explain by some specific blazar emission models. Although the data do not rule out SSC models, Wehrle et al. point out that the data are most likely explained by the "mirror" model of (Ghisellini \& Madau 1996). In this model the flaring region in the jet photo-ionizes nearby broad-emission-line clouds, which in turn provide low energy external seed photons that are inverse Compton-scattered to high energy $\gamma$-rays.

Recently, a model combining the ERC and SSC scenarios has been used to fit the simultaneous COMPTEL and EGRET spectra of PKS 0528+134 by Böttcher \& Collmar (1998). Figure 7 shows their fit to the gamma-ray spectrum during the high state of the source during March 1993. In their model Böttcher \& Collmar assume a spherical blob filled with ultrarelativistic pair plasma which is moving out along an existing jet structure perpendicular to an accretion disk around a black hole of mass $5 \times 10^{10} M_{\odot}$. They argue that the observed spectral break between COMPTEL and EGRET energy ranges can plausibly be explained by a variation of the Doppler beaming factor in the framework of a relativistic jet model for AGNs.

The EGRET results have demonstrated that in order to model the spectra of blazars it is very important to get a truly simultaneous coverage across the entire electromagnetic spectrum before, during, and after a flare in the high-energy $\gamma$-ray emission. The limited data that we have on most of the blazars prevents us from being able to distinguish between the different theoretical models, on the basis of the spectra alone. For example, both the SSC and ERC models have been shown to reproduce the multiwavelength spectrum of 3C 279 rather well (Hartman et al. 1996b; Maraschi, Ghisellini 




Figure 6. Radio to $\gamma$-ray energy distribution of 3C 279 in low (open circles) and flaring state (filled circles) in 1996 January-February (Wehrle et al. 1997).

\& Celotti 1992; Ghisellini \& Maraschi 1996). The SSC model was similarly found to fit the multiwavelength spectrum of PKS 0528+134 during the March 1993 flare reasonably well (Mukherjee et al. 1996). The low-state data of PKS 0528+134 (Aug 1994) was fit well with the ERC model, as demonstrated by Sambruna et al. (1997). The SSC, ERC, and PIC models have all been shown to fit the multiwavelength spectrum of $3 \mathrm{C} 273$ well (von Montigny 1997).

The differences between the $\gamma$-ray variability properties of BL Lac objects and FSRQs can be explained in light of the model of Ghisselini and Madau (GM) (1996). In their model, soft photons from the jet are reprocessed by broad line region (BLR) clouds. Subsequently, these soft photons are emitted back into the jet where they scatter off of electrons in relativistically moving "blobs" to create high-energy $\gamma$-rays. Since BL Lac objects generally have very weak emission lines, it may be that they have much less BLR gas available for reprocessing soft photons. If there is an initial outburst of soft photons created via the synchrotron process in a jet "blob," then BL Lac objects can still create $\gamma$-rays via the SSC process, though perhaps with lower amplitude than $\gamma$-rays created via the GM model. (This effect is somewhat dependent on adjustable model parameters.) In this scenario, BL Lac objects may undergo several SSC outbursts which fail to reach the EGRET detection threshold, thus giving the appearance that BL Lac objects as a class experience less dramatic and less frequent $\gamma$-ray flares. The general properties of the low frequency outbursts of BL Lac objects, 


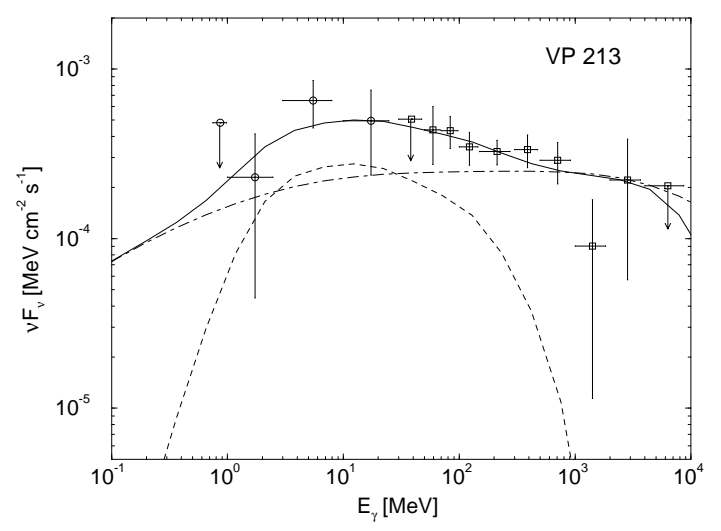

Figure \%. Fit to the $\gamma$-ray (COMPTEL and EGRET) spectrum of PKS $0528+134$ in its high state in VP 213. See Böttcher \& Collmar (1998) for fit parameters.

however, would be very similar to that of the FSRQs.

In order to achieve a better understanding of the emission mechanisms of $\gamma$-rays from blazars a study of the correlated short time scale $(\sim 1-3$ days $)$ $\gamma$-ray variations with those at other frequency bands is needed. Since the predictions of time delays between the flux changes at various frequencies are different for the individual models for both the seed photons and the nature of the inner jet, this method could provide a means to discriminate between the different models. The differences in the model predictions are discussed in more detail by Marscher et al. (1995). Gamma-ray variability in the different models may have different impacts on the spectral behavior during the build-up and decline of an outburst. Studying the short-timescale behavior and looking for spectral changes while following a complete outburst may be the key to pin down the basic emission mechanisms.

\section{Summary}

In conclusion, the EGRET results have shown the importance of the $\gamma$ ray window on blazars. The high luminosities and strong time variability observed have pushed theoretical models to emphasize relativistic jets of particles seen at small angles to the line of sight. The EGRET observations have established that the $\gamma$-ray window is critical for understanding the properties of blazars. Future observations with CGRO and successor $\gamma$ ray observatories like INTEGRAL and GLAST should play a key role in resolving the physics of these powerful sources. 
The author presents this work on behalf of the EGRET Team and acknowledges contributions from D. L. Bertsch, S. D. Bloom, B. L. Dingus, J. A. Esposito, C. E. Fichtel, R. C. Hartman, S. D. Hunter, G. Kanbach, D. A. Kniffen, Y. C. Lin, H. A. Mayer-Hasselwander, L. M. McDonald, P. F. Michelson, C. von Montigny, A. Mücke, P. L. Nolan, M. Pohl, O. Reimer, E. Schneid, P. Sreekumar, and D. J. Thompson. The author would particularly like to thank P. Sreekumar for critical comments on the draft. The author also acknowledges support from NASA Grant NAG5-3696.

\section{References}

Becker, P. A. \& Kafatos, M. 1993, in: Proceedings of the 2nd COMPTON Symposium, College Park, MD 1993, AIP Conference Proc. No. 304, eds: C. E. Fichtel, N. Gehrels, \& J. P. Norris, pg. 620

Bertsch, D. L., et al. 1989, Proc. of the Gamma Ray Observatory Science Workshop, ed. W. N. Johnson, 2, 52

Blandford, R. D. \& Königl, A. 1979, ApJ, 232, 34

Blandford, R. D. \& Levison, A. 1995, ApJ, 441, 79

Bloom, S. D. \& Marscher, A. P. 1996, ApJ, 461, 657

Bloom, S. D., et al. 1997, ApJ, 490, L145

Böttcher, M. \& Collmar, W. 1998, A\&A, 329, L57

Chiang, J. \& Mukherjee, R. 1998, ApJ, 496, 752

Collmar, W., et al. 1997, A\&A, 328, 33

Dermer, C. D. \& Schlickeiser, R. 1994, ApJS, 90, 945

Esposito, J. A., et al. 1998, in preparation

Ghisellini, G. \& Madau, P. 1996, MNRAS, 280, 67

Ghisellini, G. \& Maraschi, L. et al. 1996, "Blazar Continuum Variability," A. S. P. Conf. Series Vol. 110, pg 436

Hartman, R. C., et al. 1993, ApJ, 407, L41

Hartman, R. C., et al. 1996a, "Blazar Continuum Variability," A. S. P. Conf. Series Vol. 110, pg 333

Hartman, R. C., et al. 1996b, ApJ, 461, 698

Hartman, R. C., et al. 1997, Proc. of the Fourth Compton Symposium, eds. C. D. Dermer, M. S. Strickman, \& J. D. Kurfess, CP410, 307

Hartman, R. C., et al. 1998, ApJ, submitted

Hughes, E. B., et al. 1980, IEEE Trans. Nucl. Sci., NS-27, 364

Hunter, S. D., et al. 1993, ApJ, 409, 134

Hunter, S. D., et al. 1997, ApJ, 481, 205

Kanbach, G., et al. 1988, Space Sci. Rev., 49, 69

Kanbach, G., et al. 1989, Proc. of the Gamma Ray Observatory Science Workshop, ed. W. N. Johnson, 2, 1

Kniffen, D. A., et al. 1993, ApJ, 411, 133 
Mannheim, K. \& Biermann, P. L. 1989, A\&A, 221, 211

Mannheim, K. \& Biermann, P. L. 1992, A\&A, 53, L21

Maraschi, L., Ghisellini, G., \& Celotti, A. 1992, ApJ, 397, L5

Marscher, A. P., et al. 1995, PNAS, 92, 11439

Mattox, J. R., et al. 1993, ApJ, 410, 609

Mattox, J. R., et al. 1996, ApJ, 461, 396

Mattox, J. R., et al. 1997a, ApJ, 481, 95

Mattox, J. R., et al. 1997b, ApJ, 476, 692

McLaughlin, M. A., et al. 1996, ApJ, 473, 763

von Montigny, C., et al. 1995, ApJ, 440, 525

von Montigny, C., et al. 1997, ApJ, 483, 161

Mücke A., et al. 1996, IAU Symposium 175, (Dordrecht: Kluwer)

Mukherjee, R., et al. 1996, ApJ, 470, 831

Mukherjee, R., et al. 1997, ApJ, 490, 116

Pohl, M., et al. 1997, A\&A, submitted

Sambruna, R. M., et al. 1997, ApJ, 474, 639

Sikora, M., Begelman, M. C., \& Rees, M. J. 1994, ApJ, 421, 153

Sreekumar, P., et al. 1996, ApJ, 464, 628

Sreekumar, P., Stecker, F. W., \& Kappadath, S. C. 1997, Proc. of the

Fourth Compton Symposium, eds. C. D. Dermer, M. S. Strickman, \& J. D.

Kurfess, CP410, 307

Sreekumar, P., et al. 1998, ApJ, 494, 523

Stecker, F. W., \& Salamon, M. H. 1996, ApJ, 464, 600

Swanenburg, B. N., et al. 1978, Nature, 275, 298

Thompson, D. J., et al. 1993a, ApJS, 86, 629

Thompson, D. J., et al. 1995, ApJ, 101, 259

Thompson, D. J., et al. 1996, ApJS, Vol. 107, 227

Wagner, S. et al. 1995, ApJ, 454, L97

Wehrle, A. et al. 1997, ApJ, submitted 